\title{
ANALYSIS OF PATHOGENIC AND UNCERTAIN SIGNIFICANCE VARIANTS IN NINE GENES OF THE BRCA1-MEDIATED HOMOLOGOUS RECOMBINATION PATHWAY IN PATIENTS WITH SUSPECTED HEREDITARY BREAST AND OVARIAN CANCER SYNDROME IN CENTRAL BRAZIL.
}

Rebeca Mota Goveia', Paula Francinete Faustino Silva1, Thais Bomfim Teixeira', Isabela Gasparini Arraes¹, Ruffo Freitas-Júnior ${ }^{1}$, Elisângela Paula Silveira-Lacerda ${ }^{1}$

'Universidade Federal de Goiás - Goiânia (GO), Brazil.

Introduction: Breast cancer is the most frequent type of cancer in the world and the biggest cause of female deaths. About $10 \%-15 \%$ of cases are due to hereditary factors. The profile of genetic variants is still scarcely known among the Brazilian population and there are no published data for the central region of the country. Objectives: This study aimed to analyze the profile of pathogenic variants (PV) and of the ones of uncertain significance (VUS) for the RAD50, RAD51C, RAD51D, ATM, PALB2, BRIP1, BARD1 and CHEK2 genes in this population. Methods: 113 patients diagnosed with breast or ovarian cancer who met the National Comprehensive Cancer Networking criteria for hereditary breast and ovarian cancer syndrome were selected. The genes had all regions sequenced using NGS (New Generation Sequencing) and the raw data were evaluated using the Sophia DDM and IonReporter softwares. Results: A total of 3.53\% of patients had PV in the PALB2 (c.2257C>T), BARD1 (c.176_177delAG), RAD50 (c.2165dupA) or ATM (c.7913G>A) genes. Patients with pathogenic variants in ATM and PALB2 genes were diagnosed before the age of 40. Patients with pathogenic variants in the BARD1 and RAD50 genes had triple negative breast cancer before the age of 60 . The patient with a pathogenic variant in the RAD50 gene also developed ovarian cancer. It was observed that $24.77 \%$ of the patients had some VUS, $35.29 \%$ of which were in the ATM gene, and a new VUS in the CHEK2 gene (c.1151T>C), related to male breast cancer. Conclusions: These findings contribute to a better understanding of the phenotype of patients with pathogenic variants related to breast cancer in non-BRCA genes. In addition, it reveals a new pathogenic variant in the CHEK2 gene, not described in the literature, related to a case of male breast cancer. 